\title{
2-Dimensional flat curvature flow of crystals
}

\author{
DAVID G. CARABALLO ${ }^{\dagger}$ \\ Department of Mathematics, Georgetown University, Washington, DC 20057-1233, USA
}

[Received 16 February 2004 and in revised form 23 March 2005]

\begin{abstract}
In the impressive and seminal paper [5], Fred Almgren, Jean Taylor, and Lihe Wang introduced flat curvature flow in $\mathbb{R}^{n}$, a variational time-discretization scheme for (isotropic or anisotropic) mean curvature flow. Their main result asserts the Hölder continuity, in time, of these flows. This essential estimate requires a boundary regularity result, a uniform lower density ratio bound condition, which they proved for each $n \geqslant 3$. Similar estimates for Brownian flows, from important work by N. K. Yip on stochastic mean curvature flow [30], also rely on this pivotal regularity result.

In this paper, we complete this analysis for the case $n=2$ by establishing the necessary uniform lower density ratio bounds.
\end{abstract}

2000 Mathematics Subject Classification: 53C44, 49Q20, 49N60.

\section{Introduction}

The field of mean curvature flow began with Ken Brakke's pioneering paper [12], in which he used Almgren's varifolds to model surfaces. Varifolds, Radon measures over $\mathbb{R}^{n} \times G(n, m)$, are good for modeling quite general unoriented surfaces, which may be very complex topologically, as well as non-smooth (see [2] and [1]). Mean curvature flows in $\mathbb{R}^{n}$ attempt to decrease surface area, which is independent of orientation (isotropic), and so varifolds are a natural choice for the model.

Brakke's results were spectacular. He proved existence and partial regularity results, and he even established strong barrier estimates. In particular, he showed that spheres are barriers to evolving surfaces. He used powerful variational and measure-theoretic arguments, many of which were relatively new and not widely known at the time.

Several years later, Richard Hamilton, Michael Gage, Gerhard Huisken, and Matthew Grayson, in a series of remarkable papers, established several fundamental results for mean curvature flow. In [17] and [18], Gage and Hamilton showed that convex curves in the plane become asymptotically circular and smoothly shrink to a point. Grayson later showed that any embedded plane curve remains embedded under mean curvature flow and also becomes convex; it follows that embedded plane curves become asymptotically circular and shrink to a point (see [19]). Huisken [20] then extended these results by showing that convex surfaces tend toward spheres.

Their results used classical differential geometry and partial differential equations. Those early papers led to the rapid development of the extremely active field of curvature flow. Mean curvature flow has been studied in the plane and in higher dimensions, with hypersurfaces and in higher codimension, in both the isotropic and anisotropic cases, in Euclidean space and in other ambient manifolds, using classical methods of partial differential equations as well as level-set approaches or weak formulations, theoretically and computationally, for both single interface and multiple interface configurations.

\footnotetext{
${ }^{\dagger}$ E-mail: dgc3@georgetown.edu
} 
In many applications, such as crystal growth, the relevant surface energy can be highly anisotropic. Jean Taylor developed the notion of motion by weighted mean curvature, a process by which a crystal seeks to reduce its surface energy as quickly as possible, subject to some bulk constraints (see [27] for an excellent introduction). Motion by weighted mean curvature is of fundamental importance in materials science (see [28]). Mean curvature and weighted mean curvature flows necessarily develop singularities in finite time, but physical systems continue nonetheless. From a practical standpoint, therefore, it is essential to consider a generalized notion of curvature flow, one which can continue despite singularities. Several such weak formulations have been proposed, in both the PDE and measure-theoretic settings.

In the seminal paper [5], Fred Almgren, Jean Taylor, and Lihe Wang introduced flat curvature flow in $\mathbb{R}^{n}$, a variational time-discretization scheme for weighted mean curvature flow. They defined discrete flows, in which the crystal at the next time step (an $E$-minimizer) is selected by minimizing a certain surface plus bulk energy functional, (4), which itself was chosen so that the Euler-Lagrange condition gives surface normal velocity pointwise proportional to mean curvature. The limit flow, as the time step $\Delta t \rightarrow 0$, is the desired flat curvature flow. Here, the word "flat" refers to the flat semi-norm from geometric measure theory, not to the ambient spatial dimension. In [23], Frank Morgan relates the story of how Hassler Whitney [29] named the larger of his two norms the sharp norm, ||$_{\#}$, and the smaller one the flat norm, ||$_{b}$, by analogy with music.

The paper [5] represented a major advance in the modeling of time-evolution phenomena such as crystal growth. Their setting was so general that it admitted rough, realistic interfaces, having orientation and a wide variety of singularities. These interfaces could even undergo complex topological changes throughout their evolution. Moreover, their surface energy density functions may be isotropic or anisotropic, and may be non-differentiable (as is the case for many naturally occurring crystalline materials). Their paper was the first in such a general setting. Their flows take place in $\mathbb{R}^{n}$, though the results should readily extend to other ambient manifolds, such as Riemannian manifolds.

When the interfaces and surface energy density function are sufficiently regular, these flows coincide with classical flows given by partial differential equations until the latter develop singularities. As with physical systems, flat flows continue beyond the occurrence of singularities.

In [21], Stephan Luckhaus gave another nice time-discretized model for the isotropic case. In [4], Almgren and Taylor showed that flat flow, for crystalline energy density functions $\phi$, agrees with 2-dimensional crystalline curvature flow for polygonal crystals in the plane (see [10], [25], and [26]).

The flat flow model has been extended in several important ways. Almgren and Wang [6] added heat flow to the model, allowing for differing heat capacities of the crystal and its melt, as well as taking into account how the curvature of the interface affects the local freezing temperature (the Gibbs-Thomson effect). Nung Kwan Yip [30] introduced a stochastic time-stepping model, alternating minimization steps (which are the same as in the paper [5]) with stochastic perturbation steps. Robert McCann used flat flow in $\mathbb{R}^{2}$ to give a new proof that non-equilibrium convex crystals in $\mathbb{R}^{2}$ remain convex as they evolve by curvature-driven flow. In [13], the author allowed for partitions involving several crystals, with each interface having its own surface energy density function and its own mobility function.

The most important result for flat flows, $[K(t)]$, is an a priori existence and Hölder continuity estimate [5, 4.5] which guarantees that the limit flat flows $[K(s)]$ exist and satisfy

$$
\left\|\chi_{K(s)}-\chi_{K(t)}\right\|_{L^{1}}=\mathcal{L}^{n}(K(s) \div K(t)) \leqslant \Gamma|s-t|^{1 / 2},
$$

where $\div$ denotes the symmetric difference operation. 
Because of how flat flows are defined technically, $[K(s)]$ and $[K(t)]$ are actually limits of different sequences of integral currents, and the danger is that the flow will be wildly discontinuous in time, which would make the flow useless. It is essential, therefore, to establish an a priori Hölder continuity result to ensure that this does not happen.

The proof of the main Hölder continuity estimate, (1), is based on a volume inequality, [5, 4.2], which bounds $\mathcal{L}^{n}(K(s) \div K(s+\triangle t))$ from above in terms of $\theta$, an a priori lower bound to the $(n-1)$ dimensional density ratios at boundary points of $E$-minimizers. We sharpened and generalized [5, 4.2] in [14]. However, in both [5] and [14], the upper bounds for $\mathcal{L}^{n}(K(s) \div K(s+\Delta t))$ have $\theta$ in the denominator, which is one reason these lower density ratio bounds are critical. Another reason is that they imply that boundaries of $E$-minimizers have supports which are not too big (and have Hausdorff dimension $n-1$ in particular). This is important, because if the supports were to get too large then the entire evolution would degenerate.

In [5, 3.4], Almgren, Taylor, and Wang showed that boundaries of $E$-minimizers do have uniform $(n-1)$-dimensional lower density ratio bounds. Their proof of this pivotal theorem relies on estimating the mass of an integral $(n-1)$-current, $R$, in terms of the mass of its boundary, $\partial R$, an $(n-2)$-dimensional current. That step uses an isoperimetric inequality, which perforce introduces the ratio $(n-1) /(n-2)$ into steps used throughout the rest of the proof. In this way, they establish their main Hölder continuity theorem for each $n \geqslant 3$.

The uniform lower density ratio bounds are also vital for stochastic mean curvature flow. In [30], Yip states the result as in [5, 3.4] and gives the same proof for $n \geqslant 3$, citing [5]. He later uses the result several times to establish an essential and impressive uniform lower density ratio bound for Brownian flows and to establish a Hölder continuity theorem (following [5] and [21]).

For $n \geqslant 2$, uniform lower bounds on $(n-1)$-dimensional density ratios at boundary points play an important role in many contexts. For area-minimizing hypersurfaces, these density ratios are monotonic (see [16, 5.4], [23, 9.3]), and that fact is very helpful in establishing strong regularity for such surfaces.

Unfortunately, monotonicity does not hold for surface energy minimizers with general anisotropic energies ([1, 5.1]), but even in such cases one can usually establish uniform lower density ratio bounds (see [23, 9.5 and the discussion after 9.3]). In [24], Séverine Rigot establishes lower density ratio bounds in a fairly general context, while studying quasiminimal crystals. In [7], Luigi Ambrosio, Matteo Novaga, and Emanuele Paolini work in a general Minkowski space and establish regularity results, including a uniform lower density ratio bound ([7, 3.5]) for general $\omega$-minimizers.

In this paper, we establish the necessary uniform lower density ratio bounds (Theorem 6 for $E$-minimizers for the remaining case $n=2$, thus completing the analysis for both flat flow and stochastic mean curvature flow in $\mathbb{R}^{2}$.

The case $n=2$ is not trivial. While $\mathcal{H}^{1}$ almost every point on the reduced boundary of a set of finite perimeter in $\mathbb{R}^{2}$ has 1-dimensional density ratios which approach 1 as the balls shrink to a point (see [16, 4.5.6]), we need lower density ratio bounds for all points $p \in \operatorname{spt} \partial[K]$, which in general may be much larger than $\partial K$ and may even have positive $\mathcal{L}^{2}$ measure. We have to consider that there may be sequences of tiny cycles around boundary points, not just a nice, connected, smooth curve. Moreover, it is a very uniform, and not pointwise, estimate that is needed. We need to find a single $\theta$ which works all the way up to the same fixed positive radius for each point $p \in \operatorname{spt} \partial[K]$. So, $\theta$ must be independent of $p$. Also, $\theta$ must be independent of $K$ itself (except for the essential fact that $[K]$ is an $E$-minimizer), because we need to use that same $\theta$ to estimate $\mathcal{L}^{2}(K(s+\Delta t) \div K(s+2 \triangle t))$, 
and so on, for each step in the discrete flow. Furthermore, $\theta$ needs to be independent of $\Delta t$ as well, since we need to use it for discrete flows with arbitrary time step.

We first briefly describe the formal setting. Following Almgren, Taylor, and Wang, we use the integral and rectifiable currents of geometric measure theory. See [5], [16], and [23] for more details.

In [30], Yip also used the lower density ratio bound and a covering argument to prove a nice theorem showing that $E$-minimizers also have $n$-dimensional densities bounded uniformly from below, for each $n \geqslant 3$. In Theorem 5 , we establish the same result for $n=2$ using a new argument. It is interesting to note that, whereas he had used the $(n-1)$-dimensional density ratio bounds to establish the stronger $n$-dimensional density ratio bounds for each $n \geqslant 3$, we will do the reverse for $n=2$.

Specifically, in Theorem 5, we show that boundaries of $E$-minimizers have 2-dimensional density ratios bounded uniformly from below and from above. We then use the relative isoperimetric inequality to prove Theorem 6 , which establishes the necessary 1-dimensional density ratio bounds so that flat flows in $\mathbb{R}^{2}$ will be Hölder continuous.

We state additional regularity results in Theorem 8 , and in Corollary 7 we show that boundary supports differ from reduced boundaries of minimizers by at most a set having $\mathcal{H}^{1}$ measure zero, which guarantees that boundary supports have Hausdorff dimension 1 , as desired.

Finally, in Proposition 10, we establish lower bounds on the areas of "detachable pieces" of $E$-minimizers, and we use those bounds and Theorem 6 to prove Theorem 11 a sharper version of Theorem 6 with optimal lower bounds.

I would like to thank Frank Morgan, who, when reviewing an early draft of my doctoral thesis, observed that my theorem on lower density ratio bounds for polycrystals held only for each $n \geqslant 3$; it failed to work when $n=2$ for precisely the same reason that the proof of [5, 3.4] does not work for the case $n=2$. I then gave a separate proof [13] for the case $n=2$, for crystals and polycrystals. That proof made use of connected components and worked only for the case $n=2$. By contrast, each result in the present paper, with the sole exception of Theorem 11, extends with the same proof to any $n \geqslant 2$, the only difference being that various constants depend on $n$.

I would like to thank Matteo Novaga for his helpful comments on earlier versions of this paper. It was he who first suggested I try to prove a result like Theorem 11 He also suggested alternative strategies for proving Theorem 6, including one using connected components, as in [13], and one using the general notion of perimeter introduced in [7].

\section{Some notation}

Our ambient space is $\mathbb{R}^{2}$, endowed with the usual Euclidean norm || . Throughout this paper, $\phi$ : $\mathbb{R}^{2} \rightarrow \mathbb{R}$ has all the properties of a norm, except that $\phi$ need not be even. That is, $\phi$ is an asymmetric norm (see [14]). It will represent a "surface energy integrand," or "surface energy density function." We do not assume anything else (such as smoothness) about $\phi$.

We let $B^{2}(p, r)=\left\{x \in \mathbb{R}^{2}:|x-p| \leqslant r\right\}$ represent the closed Euclidean ball in $\mathbb{R}^{2}$, with radius $r \geqslant 0$ and center $p \in \mathbb{R}^{2}$, and we let $U^{2}(p, r)=\left\{x \in \mathbb{R}^{2}:|x-p|<r\right\}$ denote its interior. We measure length and area in $\mathbb{R}^{2}$ with Hausdorff 1 -dimensional measure, $\mathcal{H}^{1}$, and with Lebesgue 2-dimensional measure, $\mathcal{L}^{2}$, respectively.

For subsets $A, B \subset \mathbb{R}^{2}$, we write $A \subset_{2} B$ (and say that $A$ is $\mathcal{L}^{2}$ almost contained in $B$ ) provided $\mathcal{L}^{2}(A \backslash B)=0 ; A \div B$, the symmetric difference, is the set $(A \backslash B) \cup(B \backslash A)$. 


\section{Flat curvature flow}

\section{$3.1 \quad$ E-minimizers}

In this paper, crystals $[K]$ are 2-dimensional integral currents associated with bounded, $\mathcal{L}^{2}$ measurable subsets $K \subset \mathbb{R}^{2}$ having finite perimeter. For such sets $K$, the characteristic function $\chi_{K}$ is a function of bounded variation. As a current, $[K]=\mathbf{t}\left(K, 1, e_{1} \wedge e_{2}\right)$, so that $[K](\varphi)=$ $\int_{K}\left\langle e_{1} \wedge e_{2}, \varphi(x)\right\rangle \mathrm{d} \mathcal{L}^{2} x$, whenever $\varphi$ is a $C^{\infty}$ differential $n$-form with compact support.

Its boundary, $\partial[K]$, is a 1-dimensional integral current, defined via the relation $(\partial[K])(\varphi)=$ $[K](\mathrm{d} \varphi)$, whenever $\varphi$ is a smooth differential 1 -form. In the notation above, we have $\partial[K]=$ $\mathcal{H}^{1}\left\llcorner\partial K \wedge * n_{K}=\mathbf{t}\left(\partial K, 1, * n_{K}\right)\right.$, where $n_{K}$ is the measure-theoretic exterior unit normal in the sense of Federer $([16,4.5 .5])$, and $*$ is the Hodge dual. Explicitly, we have $(\partial[K])(\varphi)=$ $\int_{x \in \partial K}\left\langle * n_{K}(x), \varphi(x)\right\rangle \mathrm{d} \mathcal{H}^{1} x$. This integral is well defined, because $\partial K$ is precisely the rectifiable set of points, $x$, at which $n_{K}(x)$ is defined (see [16, 4.5.6]). There are various commonly used definitions of reduced boundary for a subset $K \subset \mathbb{R}^{2}$; these differ by sets having $\mathcal{H}^{1}$ measure 0 .

We let $\mathcal{K}$ denote the collection of all such crystals, $[K]$. It is equipped with a metric, $d_{M}$, defined by $d_{M}([K],[L])=\mathcal{L}^{2}(K \div L)$. We say that a sequence, $\left\{\left[K_{i}\right]\right\}$, of crystals in $\mathcal{K}$ converges strongly (and write that $\left[K_{i}\right] \rightarrow[K]$ strongly) if there exists a subset $K \subset \mathbb{R}^{2}$ as above for which $d_{M}\left(\left[K_{i}\right],[K]\right) \rightarrow 0$ as $i \rightarrow \infty$.

If $\phi$ is an asymmetric norm, we define the $\phi$ surface energy of $[K] \in \mathcal{K}$ by

$$
S E(\partial[K])=\int_{\partial K} \phi\left(n_{K}(x)\right) \mathrm{d} \mathcal{H}^{1} x .
$$

If $\phi$ is constant on unit vectors, we say that it is isotropic; otherwise, it is anisotropic. If $\phi$ is the Euclidean norm, then clearly $S E(\partial[K])=\mathcal{H}^{1}(\partial K)$. We define

$$
0<\phi_{0}=\inf _{|x|=1}\{\phi(x)\} \leqslant \sup _{|x|=1}\{\phi(x)\}=\phi^{0}<\infty,
$$

and we observe that 2 implies that

$$
\phi_{0} \mathcal{H}^{1}(\partial K) \leqslant S E(\partial[K]) \leqslant \phi^{0} \mathcal{H}^{1}(\partial K)
$$

Whenever $\left[K_{0}\right]$ and $[K]$ are crystals in $\mathcal{K}$, and $\Delta t>0$, we define (as in $[5,2.6]$ )

$$
E\left(\left[K_{0}\right],[K], \triangle t\right)=S E(\partial[K])+\frac{1}{\triangle t} \int_{K_{0} \div K} \operatorname{dist}\left(x, \partial K_{0}\right) \mathrm{d} \mathcal{L}^{2} x .
$$

If $E\left(\left[K_{0}\right],[K], \triangle t\right)=\inf \left\{E\left(\left[K_{0}\right],[Q], \Delta t\right):[Q] \in \mathcal{K}\right\}$, we say that $[K]$ is an $E$-minimizer for $\left[K_{0}\right]$ over $\triangle t$. The bulk integral in (4) makes it expensive for an interface to move too far in a short time interval. The Euler-Lagrange condition looks like

$$
\text { velocity }=-\frac{\triangle(\text { surface energy })}{\triangle(\text { area })} .
$$

We note (as in [5, 3.3.1]) that, if $[K]$ is an $E$-minimizer for $\left[K_{0}\right]$ over $\Delta t$, and if $[L]$ is any other crystal in $\mathcal{K}$, then

$$
S E(\partial[K])-S E(\partial[L]) \leqslant \frac{1}{\Delta t} \int_{K \div L} \operatorname{dist}\left(x, \partial K_{0}\right) \mathrm{d} \mathcal{L}^{2} x
$$




\subsection{Weighted mean curvature flow in the smooth case}

Following [27] and [5], we will define the $\phi$ weighted mean curvature, $H_{\phi, S}(p)$, of a surface $S$ at a point $p$, in the smooth case. Suppose $S$ is a smooth (twice continuously differentiable), embedded closed curve in $\mathbb{R}^{2}$, oriented by the unit normal vector field $n(p)$. We define $H_{\phi, S}: S \rightarrow \mathbb{R}^{2}$ by setting, for each $p \in S$,

$$
H_{\phi, S}(p)=n(p) \operatorname{trace}[(\operatorname{Hessian}(\phi))(n(p)) \circ(\operatorname{Hessian}(R))(p)]=n(p) h_{\phi, S}(p),
$$

where $R$ is any $C^{2}$ real-valued function defined in some neighborhood $U$ of $p$ in $\mathbb{R}^{2}$, for which

$$
S \cap U=R^{-1}(0) \quad \text { and } \quad(\nabla R)(p)=-n(p) .
$$

For example, if $S=\partial B^{2}(0, r)$, we could use $R(x, y)=\left(r^{2}-x^{2}-y^{2}\right) / 2 r$ and compute $H_{\phi, S}(p)=$ $-(1 / r) p$, whenever $p \in \partial B^{2}(0, r)$.

Suppose $g$ is a $C^{1}$ vector field on $\mathbb{R}^{2}$, and define, for each $t \geqslant 0$ and for each $x \in \mathbb{R}^{2}$, the deformation $G_{t}(x)=x+\operatorname{tg}(x)$. We note that $G_{0}(\cdot)$ is the identity transformation on $\mathbb{R}^{2}$, that $G_{t}$ is a diffeomorphism for all sufficiently small values of $t$, and that $\left.\frac{\partial}{\partial t} G_{t}(x)\right|_{t=0}=g(x)$, so that $g$ is the initial velocity vector field. If $S=\partial K$ for some bounded, $\mathcal{L}^{2}$ measurable subset $K \subset \mathbb{R}^{2}$ having finite perimeter, then we have

$$
\begin{aligned}
-\left.\frac{\mathrm{d}}{\mathrm{d} t} S E\left(G_{t}(\partial K)\right)\right|_{t=0} & =\int_{\partial K}\left[g(p) \cdot n_{K}(p)\right] h_{\phi, \partial K}(p) \mathrm{d} \mathcal{H}^{1} p \\
\left.\frac{\mathrm{d}}{\mathrm{d} t} \mathcal{L}^{2}\left(G_{t}(K)\right)\right|_{t=0} & =\int_{\partial K}\left[g(p) \cdot n_{K}(p)\right] \mathrm{d} \mathcal{H}^{1} p .
\end{aligned}
$$

Intuitively, then, $h_{\phi, \partial K}(p)$ is the infinitesimal rate of decrease of surface energy with respect to area at $p$, and so (5) takes the form velocity $=\phi$ weighted mean curvature, as desired. Therefore, we see that the energy functional $E$ has been chosen so as to approximate motion by $\phi$ weighted mean curvature at each time step. This heuristic argument can be made more precise, and, indeed, Almgren, Taylor, and Wang showed that, in the smooth case, the limit flat curvature flows agree with PDE flows until the latter develop singularities ([5]).

\subsection{Flat curvature flows}

The following is the main existence and Hölder continuity theorem for flat curvature flow in $\mathbb{R}^{n}$, for $n \geqslant 2$.

THEOREM 1 (Existence and Hölder continuity of flat flows [5, 2.6, 4.4, 4.5])

(a) Suppose $[K(0)] \in \mathcal{K}$, with $\mathcal{L}^{n}(\operatorname{spt}(\partial[K(0)]))=0$.

(b) Define discrete flows, $\left[K_{j}(t)\right]$, with time step $\triangle t_{j}=2^{-j}$, for all positive integers $j$ as follows: for each integer $j \geqslant 1$,

(b.1) set $\left[K_{j}(0)\right]=[K(0)]$,

(b.2) for each integer $k \geqslant 0$, let $\left[K_{j}\left(k \Delta t_{j}+\triangle t_{j}\right)\right]$ be an $E$-minimizer for $\left[K_{j}\left(k \triangle t_{j}\right)\right]$ over $\triangle t_{j}$,

(b.3) define $\left[K_{j}(t)\right]$ for all $t \geqslant 0$ by setting $\left[K_{j}(t)\right]=\left[K_{j}\left(k \Delta t_{j}+\Delta t_{j}\right)\right]$ whenever $k \Delta t_{j}<t \leqslant$ $(k+1) \triangle t_{j}$

(b.4) suppose $N$ is a positive integer for which $\Delta t=N \Delta t_{j} \leqslant 1$. 
(c) Suppose there exists a constant $\theta$ (a uniform lower density ratio bound) such that, for each $j \geqslant 1, k \geqslant 0$, and $p \in \operatorname{spt} \partial\left[K_{j}\left(k \triangle t_{j}\right)\right]$,

$$
\frac{\mathcal{H}^{n-1}\left(\partial K_{j}\left(k \triangle t_{j}\right) \cap B^{n}(p, r)\right)}{r^{n-1}} \geqslant \theta \quad \text { for } 0<r \leqslant \Delta t^{1 / 2} .
$$

Then there is a subsequence $i(1), i(2), i(3), \ldots$, of $1,2,3, \ldots$, and for each $t \geqslant 0$ there exist crystals $[K(t)] \in \mathcal{K}$ (we call $[K(t)]$ a flat flow) such that

$$
d_{M}\left([K(t)],\left[K_{i(j)}(t)\right]\right) \rightarrow 0
$$

locally uniformly in time $t$ as $j \rightarrow \infty$.

Moreover, there is a constant $\Gamma=\Gamma(\theta, \phi, n, S E([K(0)])) \in(0, \infty)$ such that

$$
d_{M}([K(s)],[K(s+\triangle t)]) \leqslant \Gamma \triangle t^{1 / 2}
$$

whenever $s \in[0, \infty)$ and $\Delta t \in[0,1]$.

REMARK 2 The existence of $E$-minimizers in hypothesis (b.2) above follows from a lowersemicontinuity/compactness argument given in [5].

The proof of existence of flat flows given in [5] holds for each $n \geqslant 2$, whereas the proof in [5] of the central Hölder continuity estimate (7) holds only for each $n \geqslant 3$. Specifically, Almgren, Taylor, and Wang showed that hypotheses (a) and (b) imply hypothesis (c) when $n \geqslant 3$, and that (a), (b), and (c) together imply the existence and Hölder continuity asserted in the conclusion of the theorem above.

Our main goal is to show that hypotheses (a) and (b) imply hypothesis (c) when $n=2$ as well. By the remarks above, it will then follow that the pivotal Hölder continuity inequality (7) holds when $n=2$.

REMARK 3 In [5], the uniform lower density ratio bound is given for $0<r \leqslant \Delta t$, rather than up to radius $\Delta t^{1 / 2}$. Also, their published Hölder continuity estimate involves exponent $1 /(n+1)$, rather than 1/2. However, Almgren, Taylor, and Wang had discovered these stronger results, which they mentioned in a brief remark inserted into [5] shortly before publication.

Because of how the flow $[K(t)]$ is constructed, the main Hölder estimate (7) is essential in order to preclude the evolution $[K(t)]$ from being wildly discontinuous. $[K(1 / 2)]$ is defined by showing there exists some subsequence $i_{1}(1), i_{1}(2), i_{1}(3), \ldots$ of $1,2,3, \ldots$ for which $\left\{\left[K_{i_{1}(j)}(1 / 2)\right]\right\}$ converges as $j \rightarrow \infty$. To show $[K(t)]$ exists for some other dyadic time, $t$ (i.e., $t=k 2^{-j}$ for some integers $k \geqslant 0, j \geqslant 1$ ), we find a subsequence $i_{2}(1), i_{2}(2), i_{2}(3), \ldots$ of $i_{1}(1), i_{1}(2), i_{1}(3), \ldots$ for which $\left\{\left[K_{i_{2}(j)}(t)\right]\right\}$ also converges as $j \rightarrow \infty$.

We continue to take subsequences of subsequences (and so on) until this Cantor-like argument yields a limit flow, $[K(t)]$, defined for non-negative dyadic $t$ (a countable, dense set of times). That is, we conclude that there exists a subsequence $i(1), i(2), i(3), \ldots$ of $1,2,3, \ldots$ such that $d_{M}\left([K(t)],\left[K_{i(j)}(t)\right]\right) \rightarrow 0$ as $j \rightarrow \infty$ for each dyadic $t \geqslant 0$.

Even if $s$ and $t$ are non-negative dyadic numbers which are very close together, a priori, $d_{M}([K(s)],[K(t)])=\mathcal{L}^{2}(K(s) \div K(t))$ may be quite large, because $[K(s)]$ and $[K(t)]$ were defined by using limits of different subsequences. It is very important, then, that we be able to establish a Hölder continuity estimate such as $[7$, , so that $[K(t)]$ will constitute a reasonable evolution. 


\section{Regularity results}

To estimate the bulk term in (4) from above, we will need an estimate on how far points on $\partial K$ can be from $\partial K_{0}$, if $[K]$ is an $E$-minimizer for $\left[K_{0}\right]$ over $\Delta t$. The following proposition ([5, 5.4]) gives precisely this estimate (also, see [30, 3.0.3]). In general, the constant $C=C_{\phi}$ does also depend on the ambient space dimension, $n$, but we fix $n=2$.

Proposition 4 (An upper bound on how far an interface can move) Suppose $[K]$ is an $E$-minimizer for $\left[K_{0}\right]$ over $\triangle t$. Then there exists a constant, $C=C_{\phi}$, depending only on $\phi$, for which

$$
\operatorname{dist}\left(p, \partial K_{0}\right) \leqslant C_{\phi} \Delta t^{1 / 2}
$$

whenever $[K]$ is an $E$-minimizer for $\left[K_{0}\right]$ over $\Delta t$ and $p \in \partial K$.

We now show that boundaries of 2-dimensional E-minimizers cannot be too "pointy" in a measure-theoretic sense.

THEOREM 5 (Uniform 2-dimensional lower density ratio bounds for $E$-minimizers) Suppose $[K]$ is an $E$-minimizer for $\left[K_{0}\right]$ over $\Delta t$. Then there exists a constant $\mu>0$ such that, for all $p \in \operatorname{spt} \partial[K]$, we have

$$
\min \left\{\mathcal{L}^{2}\left(K \cap B^{2}(p, R)\right), \mathcal{L}^{2}\left(\left(\mathbb{R}^{2} \backslash K\right) \cap B^{2}(p, R)\right)\right\} \geqslant \mu R^{2} \quad \text { for each } 0<R \leqslant \Delta t^{1 / 2} .
$$

Proof. If the measure $\mathcal{L}^{2}\llcorner K$ has density ratios bounded from below at each point of $\partial K$ up to some fixed positive radius, then it follows that $\mathcal{L}^{2}\llcorner K$ also has density ratios bounded from below at each point of spt $\partial[K]$, up to the same radius. Therefore, it suffices to consider each $p \in \partial K$.

Let $Q=K \cap B^{2}\left(p, \triangle t^{1 / 2}\right)$. Whenever $r \in\left(0, \triangle t^{1 / 2}\right)$, we let $Q_{r}=K \cap B^{2}(p, r)$ and $V(r)=$ $\mathbf{M}\left(\left[Q_{r}\right]\right)=\mathcal{L}^{2}\left(Q_{r}\right)$. Then $V^{\prime}(r)$ exists for $\mathcal{L}^{1}$ almost every $r>0$, since $V(r)$ is non-decreasing. Also, $V(r)>0$ since $p \in \partial K$. Let $L_{r}=K \backslash Q_{r}$, and consider the comparison crystal [ $\left.L_{r}\right]$. Let $d(x)=\operatorname{dist}\left(x, \partial K_{0}\right)$ and $\triangle S E=S E(\partial[K])-S E\left(\partial\left[L_{r}\right]\right)$. Then, since $[K]$ is an $E$-minimizer for $\left[K_{0}\right]$ over $\triangle t$ and $r \leqslant \Delta t^{1 / 2}$,

$$
\Delta S E \leqslant \frac{\int_{K_{0} \div L_{r}} d(x) \mathrm{d} \mathcal{L}^{2}-\int_{K_{0} \div K} d(x) \mathrm{d} \mathcal{L}^{2}}{\Delta t} \leqslant \frac{\int_{x \in Q_{r}} d(x) \mathrm{d} \mathcal{L}^{2}}{\Delta t} \leqslant \frac{C_{\phi}+1}{\Delta t^{1 / 2}} V(r) .
$$

Next, we will estimate $\triangle S E$ from below, using results on slicing of integral currents by Lipschitz functions ([16, 4.3], [5, 3.1.8]). Define $\rho(x)=|x-p|$, and note that Lip $\rho=1$. It follows that, for $\mathcal{L}^{1}$ almost every $r>0$, the slice $C_{r}=\langle[Q], \rho, r\rangle$ is an integral 1-current, with $\mathbf{M}\left(C_{r}\right) \leqslant V^{\prime}(r)$ and

$$
\partial\left([Q]\llcorner\{x: \rho(x)<r\})=C_{r}+\partial\left[Q_{r}\right]\llcorner\{x: \rho(x)<r\} .\right.
$$

We let $m(r)=\mathbf{M}\left(\partial\left[Q_{r}\right]\llcorner\{x: \rho(x)<r\})\right.$. The isoperimetric inequality implies that

$$
V^{\prime}(r)+m(r) \geqslant \mathbf{M}\left(C_{r}\right)+m(r) \geqslant 2 \sqrt{\pi} V(r)^{1 / 2} .
$$

This estimate, (9), and (3) imply that

$$
\triangle S E \geqslant \phi_{0} m(r)-\phi^{0} \mathbf{M}\left(C_{r}\right) \geqslant \phi_{0}\left(2 \sqrt{\pi} V(r)^{1 / 2}-V^{\prime}(r)\right)-\phi^{0} V^{\prime}(r) .
$$


We combine 8 and $(11)$ to get

$$
\left(\phi^{0}+\phi_{0}\right) V^{\prime}(r) \geqslant 2 \phi_{0} \sqrt{\pi} V(r)^{1 / 2}-\frac{C_{\phi}+1}{\Delta t^{1 / 2}} V(r) .
$$

For sufficiently small values of $r, V(r)$ will be negligible compared to $V(r)^{1 / 2}$. We now require that $r \in\left(0, \frac{\phi_{0}}{C_{\phi}+1} \Delta t^{1 / 2}\right)$. It follows that

$$
V(r)=V(r)^{1 / 2} V(r)^{1 / 2} \leqslant V(r)^{1 / 2} \sqrt{\pi r^{2}}<V(r)^{1 / 2} \sqrt{\pi}\left(\frac{\phi_{0}}{C_{\phi}+1}\right) \Delta t^{1 / 2},
$$

and so

$$
2 \phi_{0} \sqrt{\pi} V(r)^{1 / 2} \geqslant \phi_{0} \sqrt{\pi} V(r)^{1 / 2}+\frac{C_{\phi}+1}{\Delta t^{1 / 2}} V(r)
$$

(12) then becomes

$$
\left(\phi^{0}+\phi_{0}\right) V^{\prime}(r) \geqslant \phi_{0} \sqrt{\pi} V(r)^{1 / 2} .
$$

We therefore have

$$
\frac{\mathrm{d}}{\mathrm{d} r}\left[V(r)^{1 / 2}\right]=\frac{1}{2} \frac{V^{\prime}(r)}{V(r)^{1 / 2}} \geqslant \frac{1}{2} \frac{\phi_{0}}{\phi^{0}+\phi_{0}} \sqrt{\pi},
$$

and this holds (as noted above) for $\mathcal{L}^{1}$ almost every $r \in\left(0,\left[\min \left\{1, \frac{\phi_{0}}{C_{\phi}+1}\right\}\right] \triangle t^{1 / 2}\right)$.

For any $s \in\left(0,\left[\min \left\{1, \frac{\phi_{0}}{C_{\phi}+1}\right\}\right] \triangle t^{1 / 2}\right]$, we can integrate $[14$ from $r=0$ to $s$ and square both sides to get

$$
V(s) \geqslant \frac{\pi}{4}\left(\frac{\phi_{0}}{\phi^{0}+\phi_{0}}\right)^{2} s^{2} .
$$

If $\min \left\{1, \frac{\phi_{0}}{C_{\phi}+1}\right\}=1$, then we are done since for that case we have established uniform lower density ratio bounds up to radius $\Delta t^{1 / 2}$, with $\mu=\frac{\pi}{4}\left(\frac{\phi_{0}}{\phi^{0}+\phi_{0}}\right)^{2}$. If $\frac{\phi_{0}}{C_{\phi}+1}<1$, we let $R_{0}=\frac{\phi_{0}}{C_{\phi}+1} \Delta t^{1 / 2}$. For each $R \in\left[R_{0}, \Delta t^{1 / 2}\right]$, we have

$$
\frac{V(R)}{R^{2}} \geqslant \frac{V\left(R_{0}\right)}{R_{0}^{2}} \frac{R_{0}^{2}}{R^{2}} \geqslant \frac{\pi}{4}\left(\frac{\phi_{0}}{\phi^{0}+\phi_{0}}\right)^{2}\left(\frac{\phi_{0}}{C_{\phi}+1}\right)^{2}
$$

Combining these cases, we see that, for each $R \in\left(0, \Delta t^{1 / 2}\right]$,

$$
\frac{V(R)}{R^{2}} \geqslant \frac{\pi}{4}\left(\frac{\phi_{0}}{\phi^{0}+\phi_{0}}\right)^{2} \min \left\{1,\left(\frac{\phi_{0}}{C_{\phi}+1}\right)^{2}\right\}=\mu .
$$

To establish the lower bounds on the density ratios of $\mathbb{R}^{2} \backslash K$ at $p$, we can proceed using the same steps, with crystals $\left[K_{0}\right],[K]$, and $\left[L_{r}\right]$ replaced by $\left[K_{0}\right],[K]$, and $\left[L_{r}^{\prime}\right]$, where $Q_{r}^{\prime}=\left(\mathbb{R}^{2} \backslash K\right)$ $\cap B^{2}(p, r)$ and $L_{r}^{\prime}=K \cup Q_{r}^{\prime}$.

As noted above, the following theorem is the key result needed to establish Hölder continuity of flat flows in the case $n=2$. 
THEOREM 6 (Uniform 1-dimensional lower density ratio bounds for $E$-minimizers) Suppose [ $K$ ] is an $E$-minimizer for $\left[K_{0}\right]$ over $\Delta t$. Then there exists a constant $\theta>0$, constructed in the proof, such that for each $p \in \operatorname{spt} \partial[K]$ we have

$$
\frac{\mathcal{H}^{1}\left(\partial K \cap B^{2}(p, r)\right)}{r} \geqslant \theta \quad \text { for each } 0<r \leqslant \Delta t^{1 / 2} .
$$

Proof. Fix $p \in \operatorname{spt} \partial[K], 0<r \leqslant \Delta t^{1 / 2}$. The relative isoperimetric inequality (see, for example, $[15,5.6 .2])$ guarantees that there exists an absolute constant $\lambda>0$ such that

$$
\mathcal{H}^{1}\left(\partial K \cap B^{2}(p, r)\right) \geqslant \lambda \min \left\{\mathcal{L}^{2}\left(K \cap B^{2}(p, r)\right), \mathcal{L}^{2}\left(\left(\mathbb{R}^{2} \backslash K\right) \cap B^{2}(p, r)\right)\right\}^{1 / 2} .
$$

Theorem 5 then implies $\mathcal{H}^{1}\left(\partial K \cap B^{2}(p, r)\right) \geqslant \lambda \mu^{1 / 2} r$.

COROLlary 7 (Dimension of boundary support of $E$-minimizers) Suppose $[K]$ is an $E$-minimizer for $\left[K_{0}\right]$ over $\triangle t$. Then

(1) $\mathcal{H}^{1}(\overline{\partial K} \div \partial K)=\mathcal{H}^{1}(\operatorname{spt} \partial[K] \div \partial K)=0$.

(2) $\overline{\partial K}$ and spt $\partial[K]$ each have Hausdorff dimension one.

Proof. As noted in [5, 3.1.3], Theorem 6 implies (1), because of [16, 2.10.19(4) and 2.10.6]. (2) follows from (1) since $\partial K$ has Hausdorff dimension one.

Theorem 6 and Corollary 7 show that boundary supports of $E$-minimizers cannot develop elaborate filigree structures. Conclusion (2) of Corollary 7 is essential in order to define the discrete flows, since we need

$$
\mathcal{L}^{n}\left(\operatorname{spt} \partial\left[K_{j}\left(k \triangle t_{j}\right)\right]\right)=0
$$

for each $k \geqslant 0, j \geqslant 1$. We start by supposing $(15)$ to be true for $k=0$, for each $j \geqslant 1$ (see Theorem 1, hypotheses (a) and (b.1)). Corollary 7 ensures that the $E$-minimizers will continue to satisfy those hypotheses, and so (15) will hold for each $k \geqslant 0, j \geqslant 1$, as required. Finally, we note that $E$-minimizers satisfy even stronger regularity conditions.

THEOREM 8 (Additional regularity of $E$-minimizers) Suppose $[K]$ is an $E$-minimizer for $\left[K_{0}\right]$ over $\Delta t$. Then

(1) $([5,3.5]) \quad$ The current $\partial[K]$ is a $(\phi, w, \delta)$ minimal current in the sense of Bombieri $([11])$.

(2) $([5,3.6])$ If $\phi$ is even, spt $\partial[K]$ is a $(\phi, \varepsilon, \delta)$ minimal set in the sense of Almgren ([3]).

(3) $([5,3.10])$ If $\phi$ is three times Hölder continuously differentiable except at the origin, and if $\phi$ is elliptic (i.e., the restriction of $\phi$ to any arc-length parametrized line in $\mathbb{R}^{2}$ not containing the origin has strictly positive second derivatives), then spt $\partial[K]=\partial K$ is a 1 -dimensional submanifold of $\mathbb{R}^{2}$ of class 2 .

\section{Sharp density ratio bounds}

In this section, we use Theorem 6 to show that the 1-dimensional density ratios of $E$-minimizers are in fact uniformly bounded from below by 2 , up to a fixed radius depending only on $\phi$ and on $\triangle t$. We first introduce a notion, that of a "detachable piece," which will be quite helpful in the proof of our theorem. 
Definition 9 Suppose $X$ is an $\mathcal{L}^{2}$ measurable subset of $\mathbb{R}^{2}$ having finite perimeter. $D$ is called a detachable piece of $X$ provided

(1) $D$ is an $\mathcal{L}^{2}$ measurable subset of $\mathbb{R}^{2}$, with $\mathcal{L}^{2}(D)>0$, and with finite perimeter.

(2) $D$ is a subset of $X$, except possibly for a set having $\mathcal{L}^{2}$ measure zero.

(3) $\partial D \cup \partial(X \backslash D)$ is equal to $\partial X$, except possibly for a set having $\mathcal{H}^{1}$ measure zero.

(4) For $\mathcal{H}^{1}$ almost every $x \in \partial D$, we have $n_{D}(x)=n_{X}(x)$, and for $\mathcal{H}^{1}$ almost every $x \in \partial(X \backslash D)$ we have $n_{X \backslash D}(x)=n_{X}(x)$.

(5) spt $\partial[D] \cap \operatorname{spt} \partial[X \backslash D]$ has $\mathcal{H}^{1}$ measure zero.

If $X$ has no non-trivial detachable pieces, we say that $X$ is attached.

We note that a connected component of $X$ may consist of several detachable pieces of $X$, and a detachable piece of $X$ may consist of several connected components of $X$. Moreover $X$ may be attached or not, regardless of whether it is connected or not. Also, it follows immediately from the definitions that

$$
S E(\partial[D])+S E(\partial[X \backslash D])=S E(\partial[X])
$$

whenever $D$ is a detachable piece of $X$. The following proposition estimates from below the area of a detachable piece. Since connected components separated by positive distance are detachable pieces as well, the proposition applies, in particular, to pairwise disjoint closed connected components of a minimizer or its complement.

Proposition 10 (Area bounds for detachable pieces of $E$-minimizers) Suppose $[K]$ is an $E$ minimizer for $\left[K_{0}\right]$ over $\triangle t$. Suppose $D$ is a detachable piece of $K$ (or of $\mathbb{R}^{2} \backslash K$ ). Then

(1) If $c$ is any number such that $\operatorname{dist}(x, \partial K) \leqslant c \Delta t^{1 / 2}$ for $\mathcal{L}^{2}$ almost every $x \in D$, then

$$
\mathcal{L}^{2}(D) \geqslant \pi\left(\frac{2 \phi_{0}}{c+C_{\phi}} \Delta t^{1 / 2}\right)^{2} .
$$

(2) If $\lambda$ is the unique positive real number for which $\lambda=\frac{2 \phi_{0}}{\lambda+C_{\phi}}$, then

$$
\mathcal{L}^{2}(D) \geqslant \pi\left(\lambda \triangle t^{1 / 2}\right)^{2} \text {. }
$$

Proof. First, suppose $D$ is a detachable piece of $K$, and let $L=K \backslash D$. Using the isoperimetric inequality, $\sqrt{3}, \sqrt{16}, \sqrt{6}$, and Proposition 4 (noting that $\operatorname{dist}\left(x, \partial K_{0}\right) \leqslant \operatorname{dist}(x, \partial K)+C_{\phi} \triangle t^{1 / 2}$ ), we estimate

$$
\begin{aligned}
2 \phi_{0} \sqrt{\pi} \sqrt{\mathcal{L}^{2}(D)} & \leqslant \phi_{0} \mathcal{H}^{1}(\partial D) \leqslant S E(\partial[D])=S E(\partial[K])-S E(\partial[K \backslash D]) \\
& \leqslant \frac{1}{\Delta t} \int_{D} \operatorname{dist}\left(x, \partial K_{0}\right) \mathrm{d} \mathcal{L}^{2} x \\
& \leqslant \frac{1}{\Delta t} \int_{D}\left(c \Delta t^{1 / 2}+C_{\phi} \Delta t^{1 / 2}\right) \mathrm{d} \mathcal{L}^{2} x=\frac{1}{\triangle t^{1 / 2}} \mathcal{L}^{2}(D)\left(c+C_{\phi}\right) .
\end{aligned}
$$

(1) now follows by elementary algebra. For $\mathcal{L}^{2}$ almost every $x \in D$, we have $\mathcal{L}^{2}\left(D \cap B^{2}(x, r)\right)>0$ for each $r>0$. Let $x$ be such a point. If $\mathcal{L}^{2}(D) \geqslant \pi \lambda^{2} \triangle t$, we are done, so consider the case where $\mathcal{L}^{2}(D)<\pi \lambda^{2} \triangle t$. For any $r \geqslant \lambda \triangle t^{1 / 2}$, we have $\mathcal{L}^{2}\left(\left(\mathbb{R}^{2} \backslash D\right) \cap B^{2}(x, r)\right)>0$, so the relative isoperimetric inequality implies that $\mathcal{H}^{1}\left(\partial K \cap B^{2}(x, r)\right)>0$, and $\operatorname{sod} \operatorname{dist}(x, \partial K) \leqslant r$. Thus, $\operatorname{dist}(x, \partial K) \leqslant \lambda \triangle t^{1 / 2}$ for $\mathcal{L}^{2}$ almost every $x \in D$, since $\lambda$ depends only on $\phi$, and so (2) follows from (1). If $D$ is a detachable piece of $\mathbb{R}^{2} \backslash K$, we proceed as above but with $L=K \cup D$. 
THEOREM 11 (Sharper 1-dimensional density ratio bounds) Suppose [K] is an $E$-minimizer for $\left[K_{0}\right]$ over $\triangle t$. Then for each $p \in \operatorname{spt} \partial[K]$ we have

$$
\frac{\mathcal{H}^{1}\left(\partial K \cap B^{2}(p, r)\right)}{r} \geqslant 2
$$

whenever $0<r<r_{0}=\delta_{\phi} \Delta t^{1 / 2}$. Here, $\delta_{\phi}=\min \left\{1, \frac{2 \phi_{0}}{1+C_{\phi}}\right\}$, where $C_{\phi}$ is the constant from Proposition 4

Proof. If the measure $\mathcal{H}^{1}\llcorner\partial K$ has density ratios bounded from below at each point of $\partial K$ up to some fixed positive radius, it follows that $\mathcal{H}^{1}\llcorner\partial K$ also has density ratios bounded from below at each point of spt $\partial[K]$, up to the same radius and with the same bounds. Therefore, it suffices to consider each $p \in \partial K$. Suppose $p \in \partial K$, and define $f(x)=|x-p|$ for $x \in \mathbb{R}^{2}$. We note that Lip $f=1$.

The remainder of the proof will be devoted to showing that $\mathcal{H}^{0}\left(\overline{\partial K} \cap \partial B^{2}(p, r)\right) \geqslant 2$ for $\mathcal{H}^{1}$ almost every $r \in\left(0, r_{0}\right)$; our result then follows immediately by applying Corollary $7(1)$ and Corollary 2.10 .11 of [16], with $f, X, Y, A$, and $m$ there replaced by $f, \mathbb{R}^{2}, \mathbb{R}, \overline{\partial K} \cap B^{2}(p, r)$, and 1 , respectively, since

$$
\mathcal{H}^{1}\left(\partial K \cap B^{2}(p, r)\right)=\mathcal{H}^{1}\left(\overline{\partial K} \cap B^{2}(p, r)\right) \geqslant \int_{0}^{r} \mathcal{H}^{0}\left(\overline{\partial K} \cap \partial B^{2}(p, y)\right) \mathrm{d} \mathcal{H}^{1} y \geqslant 2 r .
$$

[5. 3.1.8] implies that for $\mathcal{H}^{1}$ almost every $r>0$ we have $\mathcal{H}^{1}\left(\partial K \cap \partial B^{2}(x, r)\right)=0$, and the slice current $\langle\partial[K], f, r\rangle$ is a zero-dimensional integral current. We restrict attention to such $r$ 's and note that, if $\mathcal{H}^{0}\left(\overline{\partial K} \cap \partial B^{2}(p, r)\right)$ equals 0 or 1 , then the set

$$
S=\partial B^{2}(p, r) \backslash\left(\overline{\partial K} \cap \partial B^{2}(p, r)\right)
$$

is connected. Because $\overline{\partial K}$ and $\partial B^{2}(p, r)$ are closed, $S=A \cup B$, where

$$
\begin{aligned}
& A=\left\{x \in S: U^{2}(x, R) \subset_{2} K \text { for some } R>0\right\}, \\
& B=\left\{x \in S: U^{2}(x, R) \subset_{2} \mathbb{R}^{2} \backslash K \text { for some } R>0\right\} .
\end{aligned}
$$

Clearly, both $A$ and $B$ are relatively open in $S$. Since they are complements, they are also relatively closed in $S$. Since $S$ is connected, it follows that either $A=\emptyset$ and $B=S$, or $A=S$ and $B=\emptyset$. In the former case, $K \cap U^{2}(p, r)$ is a detachable piece of $K$, while in the latter case $\left(\mathbb{R}^{2} \backslash K\right) \cap U^{2}(p, r)$ is a detachable piece of $\mathbb{R}^{2} \backslash K$.

We restrict $r$ so that $r<\Delta t^{1 / 2}$ and then apply Proposition 10 (1) with $c=1$ to deduce that this detachable piece must have area at least $\pi\left(\frac{2 \phi_{0}}{1+C_{\phi}} \Delta t^{1 / 2}\right)^{2}$, which is impossible since the area of any subset of $U^{2}(p, r)$ must be strictly less than $\pi r_{0}^{2}$. We conclude that $\mathcal{H}^{0}\left(\overline{\partial K} \cap \partial B^{2}(p, r)\right) \geqslant 2$ for $\mathcal{H}^{1}$ almost every $r \in\left(0, r_{0}\right)$.

\section{Conclusion}

Flat curvature flow, introduced by Almgren, Taylor, and Wang in [5], is a very powerful, versatile model for studying curvature flow, whether isotropic or non-isotropic. It is general enough to allow for singularities and topology changes, as with physical systems, and the flows agree with classical 
flows as long as the latter are non-singular. In addition, it can be used as a tool to re-prove classical results in the smooth setting, and it can even be used to provide limits to which computational flows might converge as $\Delta t \rightarrow 0$. The method can be extended to incorporate more physically interesting properties, such as heat flow and the Gibbs-Thomson effect ([6]), or randomness ([30]).

The most essential result for any flat flow model is an a priori Hölder continuity theorem (Theorem 11, first established in [5]. This result depends primarily on a regularity result-a uniform lower density ratio bound estimate-previously established for each $n \geqslant 3$ in [5] and established here using a different approach for $n=2$ in Theorem 6. Our approach has the added advantage that it works without alteration (except for constants that now depend on $n$ ) for any $n \geqslant 2$, so that it is not necessary to treat the cases $n=2$ and $n \geqslant 3$ separately. Using Theorem 6 we establish sharp lower bounds on the 1-dimensional density ratios of boundaries of $E$-minimizers, using techniques that will extend to other, related minimization problems in $\mathbb{R}^{2}$.

\section{REFERENCES}

1. Allard, W. K. On the first variation of a varifold. Ann. of Math. 95 (1972), 417-491. Zbl 0252.49028 MR 0307015

2. Almgren, F. J., JR. The Theory of Varifolds. Multilithed notes, Princeton Univ. Library (1965), 178 pages.

3. Almgren, F. J., JR. Existence and regularity almost everywhere of solutions to elliptic variational problems with constraints. Mem. Amer. Math. Soc. 165 (1976). Zbl 0327.49043 MR 0420406

4. Almgren, F. J., JR. \& TAYLOR, J. E. Flat flow is motion by crystalline curvature for curves with crystalline energies. J. Differential Geometry 42 (1995), 1-22. Zbl $0867.58020 \mid$ MR 1350693

5. Almgren, F. J., JR., TAylor, J. E., \& WAng, L. Curvature driven flows: a variational approach. SIAM J. Control Optim. 31 (1993), 387-438. Zbl 0783.35002 MR 1205983

6. Almgren, F. J., JR. \& WANG, L. Mathematical existence of crystal growth with Gibbs-Thomson curvature effects. J. Geom. Anal. 10 (2000), 1-100. Zbl 0981.74041 MR 1758583

7. Ambrosio, L., Novaga, M., \& Paolini, E. Some regularity results for minimal crystals, ESAIM Control Optim. Calc. Var. 8 (2002), 69-103. Zbl pre01967368 MR 1932945

8. Ambrosio, L. \& Soner, H. M. Level set approach to mean curvature flow in arbitrary codimension. J. Differential Geom. 43 (1996), 693-737. Zbl $0868.35046 \mid$ MR 1412682

9. Angenent, S. Some recent results on mean curvature flow. Recent Advances in Partial Differential Equations (El Escorial, 1992), RAM Res. Appl. Math. 30, Masson, Paris (1994), 1-18. Zbl 0796.35068 MR 1266199

10. Angenent, S. \& Gurtin, M. E. Multiphase thermomechanics with interfacial structure. Arch. Rat. Mech. Anal. 108 (1989), 323-391. Zbl 0723.73017 MR 1013461

11. Bombieri, E. Regularity theory for almost minimal currents. Arch. Rat. Mech. Anal. 78 (1982), 99-130. Zbl 0485.49024 MR 0648941

12. Brakke, K. A. The Motion of a Surface by its Mean Curvature. Math. Notes 20, Princeton Univ. Press, Princeton, NJ (1978) Zbl 0386.53047 MR 0485012

13. CARABAllo, D. G. A variational scheme for the evolution of polycrystals by curvature. Princeton Univ. Ph.D. thesis (1997).

14. Caraballo, D. G. Estimates on the areas of level sets of distance functions induced by asymmetric norms. Pacific J. Math. 218 (2005), 37-52.

15. Evans, L. C. \& GARIEPY, R. F. Measure Theory and Fine Properties of Functions, CRC Press (1992). Zbl 0804.28001 MR 1158660

16. Federer, H. Geometric Measure Theory, Springer (1969). Zbl 0176.00801 MR 0257325 
17. GAge, M. E. Curve shortening makes convex curves circular. Invent. Math. 76 (1984), 357-364. Zbl 0542.53004 MR 0742856

18. Gage, M. E. \& Hamilton, R. S. The heat equation shrinking convex plane curves. J. Differential Geom. 23 (1986), 69-96. Zbl 0621.53001 MR 0840401

19. Grayson, M. A. The heat equation shrinks embedded plane curves to round points. J. Differential Geom. 26 (1987), 285-314. Zbl 0667.53001 MR 0906392

20. Huisken, G. Flow by mean curvature of convex surfaces into spheres. J. Differential Geom. 20 (1984), 237-266. Zbl 0556.53001 MR 0772132

21. LuCKhaus, S. Implicit time discretization for the mean curvature flow equation. Calc. Var. Partial Differential Equations 3 (1995), 253-271. Zbl 0821.35003 MR 1386964

22. MCCAnn, R. J. Equilibrium shapes for planar crystals in an external field. Comm. Math. Phys. 195 (1998), 699-723. Zbl 0936.74029 MR 1641031

23. Morgan, F. Geometric Measure Theory: a Beginner's Guide. 3rd ed., Academic Press (2000). Zbl 0974.49025 MR 1775760

24. Rigot, S. Quasiminimal crystals with a volume constraint and uniform rectifiability. J. Math. Pures Appl. (9) 82 (2003), 1651-1695. Zbl 1034.49044 MR 2025315

25. TAYlor, J. E. Motion by crystalline curvature. Computing Optimal Geometries, J. E. Taylor (ed.), Selected Lectures in Mathematics, Amer. Math. Soc. (1991), 63-65 plus video.

26. TAYlor, J. E. Motion of curves by crystalline curvature, including triple junctions and boundary points. Differential Geometry, Proc. Sympos. Pure Math. 51, Part 1, Amer. Math. Soc. (1993), 417-438. Zbl 0823.49028 MR 1216599

27. TAYlor, J. E. Mean curvature and weighted mean curvature. Acta Metall. Mater. 40 (1992), 1475-1485.

28. TAylor, J. E., CAhn, J. W. \& Handwerker, C. A. Geometric models of crystal growth. Acta Metall. Mater. 40 (1992), 1443-1474.

29. Whitney, H. Geometric Integration Theory. Princeton Univ. Press, Princeton (1957). Zbl 0083.28204 MR 0087148

30. YIP, N. K. Stochastic motion by mean curvature. Arch. Rat. Mech. Anal. 144 (1998), 313-355. Zbl 0930.60047 MR 1656479 